\title{
Les origines de l'industrie céramique à Apt : l'apport des sources
} écrites XIVe- XVle siècles

Henri Amouric

\section{Citer ce document / Cite this document :}

Amouric Henri. Les origines de l'industrie céramique à Apt : l'apport des sources écrites XIVe- XVle siècles. In: Archéologie du Midi médiéval. Tome 4, 1986. pp. 131-134;

doi : https://doi.org/10.3406/amime.1986.1123

https://www.persee.fr/doc/amime_0758-7708_1986_num_4_1_1123

Fichier pdf généré le 16/04/2018 


\title{
Résumé
}

A l'époque moderne Apt (Vaucluse) est un centre de production céramique de grande réputation. En l'absence de données archéologiques, les recherches menées dans les sources écrites pour connaître les origines de cette activité ont révélé l'existence d'ateliers médiévaux. Tel qu'il apparaît, le cas d'Apt est à la fois banal et remarquable : les difficultés de l'approvisionnement en tuiles qui sont manifestes à la fin du XIVe siècle et au début du XVe siècle sont communes à là plupart des villes provençales.

La crise de production de l'artisanat céramique et sa disparition provisoire au tournant du XIVe siècle sont des phénomènes plus rares. Les conséquences, intervention de la communauté, appel à la main d'oeuvre extérieure n'en sont que plus intéressantes. Au total c'est un moment charnière d'un développement économique médiocre qui est décrit dans tous les aspects que les sources permettent d'aborder, événements, hommes, hiérarchies, structures.

\begin{abstract}
In modern times, Apt (Vaucluse) has a solid reputation as a centre of production ofceramics. For want ofarcheolo- gical facts, research among written sources to learn the origins of this activity have revealed the existence of medieval workshops. The case of Apt appears to be both common as well as noteworthy : the problem of supply oftiles which makes itself felt towards the end of the XIV century and at the beginning of the XV century is common to most towns of Provence.

The crisis pottery handcraft went throught and its temporary disappearrance at the turn of the XIV century however, are less common. The sequels are all the more interesting: communities stepped in, there was a callfor labour from outside. On the whole it is a crucial moment of a mediocre growth which has been described from all points ofview that sources have made available ; events, people, hierarchy, structures.
\end{abstract}




\title{
LES ORIGINES \\ DE L'INDUSTRIE CERAMIQUE A APT : L'APPORT DES SOURCES ECRITES : XIVe - XVIe SIËCLES
}

\author{
Henri AMOURIC *
}

A l'époque moderne Apt (Vaucluse) est un centre de production céramique de grande réputation. En l'absence de données archéologiques, les recherches menées dans les sources écrites pour connaître les origines de cette activité ont révélé l'existence d'ateliers médiévaux. Tel qu'il apparaît, le cas d'Apt est à la fois banal et remarquable : les difficultés de l'approvisionnement en tuiles qui sont manifestes à la fin du XIVe siècle et au début du XVe siècle sont communes à la plupart des villes provençales.

La crise de production de l'artisanat céramique et sa disparition provisoire au tournant du XIV ${ }^{c}$ siècle sont des phénomènes plus rares. Les conséquences, intervention de la communauté, appel à la main d'œuvre extérieure n'en sont que plus intéressantes. Au total c'est un moment charnière d'un développement économique médiocre qui est décrit dans tous les aspects que les sources permettent d'aborder, événements, hommes, hiérarchies, structures.

In modern times, Apt (Vaucluse) has a solid reputation as a centre of production of ceramics. For want of archeological facts, research among written sources to learn the origins of this activity have revealed the existence of medieval workshops. The case of Apt appears to be both common as well as noteworthy: the problem of supply of tiles which makes itself felt towards the end of the XIV century and at the beginning of the XV century is common to most towns of Provence.

The crisis pottery handcraft went throught and its temporary disappearrance at the turn of the XIV century howerver, are less common. The sequels are all the more interesting: communities stepped in, there was a call for labour from outside. On the whole it is a crucial moment of a mediocre growth which has been described from all points of view that sources have made available; events, people, hierarchy, structures.

En l'absence, que nous espérons toute provisoire, de résultats archéologiques probants, notre propos se limite à quelques apports des sources écrites à la connaissance des industries céramiques d'Apt depuis la fin du $\mathrm{XIV}^{\mathrm{e}}$ siècle jusqu'au début du XVI ${ }^{\mathrm{e}}$ siècle. Cette chronologie est une chronologie obligée. Elle reflète par sa date haute l'état des sources disponibles et illustre a contrario la nécessité d'une collaboration étroite avec les archéologues pour tenter de comprendre, en particulier, ce qui s'est passé antérieurement. En travaillant à ce sujet, nous nous sommes heurtés à un problème de définition: notre démarche initiale s'appliquait uniquement aux céramiques culinaires et d'ornement et ignorait tout ce qui concerne la production des céramiques architecturales. Nous en avons été en partie dissuadé par la difficulté obvie de séparer ces deux types de production qui n'étaient peut-être pas très éloignées, voire distinguées, et aussi par le constat que la céramique architecturale (en particulier les mallons) a contribué à faire la réputation d'Apt. Puisque nous nous intéressions à l'une des productions, il était logique de s'intéresser aux autres. Mais il faut au préalable émettre des réserves car la fabrication des tuiles et mallons qui est le fait des teulerii (tuiliers) pose un problème qui n'est pas spécifique à Apt, mais général dans la Provence médiévale et moderne. Les difficultés rencontrées par les communautés provençales pour s'assurer un approvisionnement régulier, donc la résidence d'un ou plusieurs tui- liers, sont une règle qui souffre des exceptions mais peu nombreuses en définitive. Il n'y a donc a priori rien d'étonnant dans les difficultés renouvelées que rencontrent les édiles aptésiens pour assurer la fourniture des tuiles et mallons nécessaires à la Ville. Les problèmes deviennent sensibles à compter des années 1383-1385. En 1380 et 1382 (1), nous avons témoignage du fonctionnement de la tuilière qui, à Apt comme dans la majorité des cas, appartient à la communauté. En 1383 (2), nous apprenons le nom du tuilier du moment, Jean Gras ou Grassi. Il se livre à une activité semble-t-il assez éloignée de celle qui devrait être la sienne puisqu'il vend un certain nombre de ruches, qu'il avait installé dans la tuilière ; est-ce pour cette raison ou une autre ? Toujours est-il qu'en 1385 (3), le conseil s'émeut de ce qu'il ne produise rien et lui ordonne de fabriquer des tuiles sous peine d'être obligé de rembourser ce que la communauté lui a offert.

C'est ici un bon exemple de l'attitude des communautés vis-à-vis des artisans tuiliers. Soucieuses de s'assurer la suffisance, elles n'hésitent pas à faire des conditions très favorables à ceux-ci pour les attirer. Le plus souvent concession gratuite, aide d'hommes pour l'extraction, la remise en état de la cabane, du four, gratifications diverses, affranchissement, etc... Le jeu des tuiliers est simple. Il s'agit de prendre le maximum quitte à ne rien donner en échange et à recommencer ailleurs. Jean Gras disparaît sans que l'on sache comment et en

* Laboratoire d’Archéologie Médiévale Méditerrranéenne, U.R.A. 6 - C.N.R.S.

(1) A.C. Apt, BB13, f०20, 13 mai 1380, et fo 102,15 janvier 1382.

(2) Cité par F. Sauve dans « Le Mercure aptésien », n 4411, 1907, 28 janvier 1383. Original perdu.

(3) A.C. Apt, BB14, fo 93, $13 \mathrm{AV}$ 1385: "Item ordinaverunt quod dicti sindici debeant requirire Johannem Grassi teulerium quod ipse ab inde inantea operatur et faciat tegulos prout dicte universitati apte de pacto tenetur. Et eo casu quo adque promittetur de pacto tenetur facere nollet restituat dicte universitati pecunia quam ab ea protterea habuit ". 
1387, c'est Rostaing Ollier ou (Pelat) tuilier de Bédoin qui prend la relève (4). A cette èpoque, la communauté cherche à lui assurer une terre pour faire ses tuiles. Le schéma est le même : la communauté achète la terre de Guill. Faraud et la concède gracieusement à Rostaing ollier (5). Nous ne savons pas grand chose de ce dernier, mais son cas est intéressant, car il est dit tuilier en 1387 , et ollier (associé à un autre potier) en 1395 (6). Nous nous trouvons ici confronté au vieux problème de la spécialisation. Il semble bien dans le cas présent comme dans d'autres que notre ollier soit concomittamment tuilier. Mais il nous est impossible de généraliser à partir de ce cas précis.

Parallèlement, nous possédons quelques indices concernant les autres productions céramiques. Nous connaissons ainsi pour la fin du XIV ${ }^{\mathrm{e}}$ siècle les potiers :

Guil. Vellat (en 1374)

Jean Jérolme 1377

ou Gironimi 1380

Rostaing Ollier (Pelat)

Michel Castoli 1395 (7).

Il existe aussi un lieu dit "Las Balmas dels oliers" mentionné à plusieurs reprises (1378-1379).

Au tournant du $X V^{e}$ siècle, la situation économique d'Apt se dégrade et se tend. Nous en avons un écho dans le conseil du 5 août 1401 qui se préoccupe depopulationem huius civitatis (8). Pour lutter contre cet état de fait, le conseil autorise le retour des débiteurs, invités à s'entendre avec leurs créanciers, sous l'égide de deux syndics-arbitres nommés. Tous les secteurs d'activité sont touchés; l'artisanat céramique n'y échappe pas.

- en 1403, ce sont deux tuiliers de Reillane qui sont affranchis de tailles communales et hébergés gracieusement par la ville à condition de faire résidence et de travailler à Apt (9).

- en 1410 , la communuté prend en charge les frais de la défense du nouveau tuilier Guillaume Genies attaqué semble-t-il du fait de la tuilerie qui lui avait été remise des mains des syndics. Nous savons dans ce cas précis ce que Guill. Genies a reçu comme gratification; c'est une somme importante: 17 florins d'or auxquels s'ajoutent 2 saumées d'annone (blé). En outre, la même année Guill. Genies entre en possession de la tuilerie nouvellement achetée par la ville, à condition d'y faire résidence (10).

- en 1412 , le conseil multiplie les démarches pour tenter de s'assurer les services d'un potier résident. La conclusion qui vient immédiatement à l'esprit est qu'il n'y a plus de potier en activité dans l'Apt de 1412 ; c'est sans doute vrai.

Dans un premier temps, le conseil fait un don de 4 florins (mais prudent s'assure la caution pesonnelle de Lazare Béranger). Puis on achète au meilleur prix, les crotes de Bertrand Faraud et le terralhum de Monet Gausii inquibus crotis operentur olle... L'achat des crottes coûte 10 florins à lui tout seul. En février, le potier s'installe officiellement et la communauté lui fait un nouveau don en argent qu'il s'engage à restituer s'il part. Il est ensuite affranchi de tailles pour 5 ans sous conditions de remboursement de l'arriéré en cas de départ (11). Nous apprenons à cette occasion son nom. Il s'appelle Vitalis (...X...), son prénom a été laissé en blanc sur la page du registre; nous ignorons son destin, toujours est-il que :

- en 1413, la communauté essaie d'attirer un potier de Bédoin en lui offrant un atelier pro operando olas et en lui promettant 10 florins pour la construction d'un four à condition qu'il y demeure (12). Ce dut être le cas car,

- en 1414, il reçoit les 10 florins pour payer son tour, le four et les portes de sa crotte. Par la suite, on lui concède les crottes qui avaient été achetées à Bertrand Faraud et en prime, on l'affranchit de tailles communales (13) pour 5 ans. Enfin en 1417 on lui remet le terralh de Monet Gausii! (14).

Il est difficile de comprendre cet acharnement de la part de la communauté, le souci de suffisance n'est peut-être pas un argument décisif. Il est possible à cette époque de s'approvisionner aux marchés extérieurs. En 1411 et 1417 , on fait venir des mesures de terre et des pichets de Tarascon (15). Bédoin tout proche compte de nombreuses fabriques. Peut-être faut-il voir le souci très moderne de maintenir une activité qui avait précédemment une certaine importance. L'artisanat est une des

(4) Ibid., id., f० $157 \vee^{\circ}, 7$ juin 1387.

(5) Id., f० 160, 25 juillet 1387: "Item ordinaverunt quod extimatores accedant extimatum terram Guillermi Faraudi de Apta necessariam rostagni Pelati teulerio pro tegulis faciendis qua extimata dicta terra ematur et emo pretium solvatur de pecunia dicte civitatis ".

(6) A.D. 84, Notaires d'Apt, étude Pondicq, Louis de Roche, n 56, 12 mars 1395.

(7) Ibid., III F5, f० 25, 20 mars 1374, original perdu. Ibid., Notaires d'Apt, Pondicq 8, cahier de 1377, 13 déc., "Johannes Gironimi olerio aptensis ». Ibid., III F5, fo 103, 3 mai 1380, « Jean Jerolme » olerius d'Apt, original perdu. Rostaing Ollier et Michel Castoli, cf. notc 6 .

(8) A.C. Apt, BB15, f० 79 .

(9) Ibid., Id., fo $138 \mathrm{v}^{\circ}, 26$ janvier 1403 : "Item eodem modo ordinaverunt quod duo teulerii rellanie sint franqui et liberi a solutione talharum si veniant hinc et moram continuram (sic) faciant in presenti civitate et ultra habeantur eis hospitium sumptibus civitatis $"$.

(10) Ibid., Id., fo $265,268 v^{\circ}, 278 v^{\circ}, 23$ février, 8 avril et 9 sept. 1410

(11) Ibid. BB16, fo 29, $29 \mathrm{v}^{\circ}, 31$ v 0,$33 ; 4$ et 13 janvier 1412,4 et 17 février 1412.4 janvier $1412:$ "Ordinaverunt quod emantur crote Bertrandus Faraudi et terralhum Monneti Gausii in quibus crotis operentur olle et quod detur justum pretium et debitum de eisdem crotis..." , 4 fév. 1412: "Ordinaverunt quod detur unus francus olerio qui nunc habitat in presenti civitate dum tamen ipse faciat suum homagium de Morando in presenti civitate et Mansionem suam faciendo in eadem et se obliget illud restituere casu quo recedetur $"$.

(12) Ibid, Id., f० $61 \mathrm{v}^{\circ}, 30$ sept. 1413 : "Ordinaverunt quod eo casu quo que olerius qui nunc est in presenti civitate de bedoyno stare voluitur et mansionem suam pertettuam facere in presenti civitate quod eidem detur hospitium per universitatem emptum pro operando olas et ulterius pro furno fiendo succuratur eidem de decem $f(1)$ orenos atri " .

(13) Ibid.. Id., fo 80, 16 juin 1414 : "Ordinaverunt quod olerio succuratur pro furno et rota ac portis in crotis universitatis fiendis de decem florenos auri..." et $f^{\circ} 34 \vee \circ, 2$ juillet 1414 .

(14) Ibid., Id., fo 152 v 0,6 juillet 1417: "Ordinaverunt quod requiritur monetus Gausii ut habeat tradere terralhum olerio... ".

(15) Ibid., Id., fo 21, 29 octobre 1411 : "Item etiam ordinaverunt quod fiat mensure terre Villa Tharasconis pro provisione duodecim tabernarum actenta quod est magnum interest est predicte universitatis", 30 sept. 1417: "Item ordinaverunt quod dictum (sic) sindici faciant facere mensuras taberne de terre et pitalphos terre in loco tharasconis et quod ea que custabunt solvantur per thesaurerium". 
composantes de la ville, il fait la ville économiquement. Les édiles aptésiens en étaient peut-être conscients. La crise de l'industrie céramique semble s'achever vers 1425. En 1424, meurt le tuilier Guill. Genies. Il lègue la tuilerie à $P$. Feraud, un savoyard, la communauté avalise l'accord et il s'associe à Guill. Gueysse pour faire des tuiles à Apt ou ailleurs (indice d'une certaine mobilité) (16).

Le restant du XVc et le début du XVIc siècle voient le redémarrage de l'activité céramique à un rythme qui devait être assez soutenu à en juger par le nombre plutôt important des potiers connus.

Il y a tout d'abord :

Bertrand Faraud en 1412 (possède des ateliers),

Monet Gausii vers 1425-1427,

Antoine Sabbatier

Pierre Badel (1427-29 (17),

Antoine Gausii (1438) (18),

Antoine David (1438-1466) (19),

Pierre Armand (1438), (procès avec la communauté en 1445), mariage en $1446(20)$,

Petrus Oliverii (1440) (21),

Antoine Galvi (1450-1451) (22),

Jean Barralier (neveu d'Antoine Gausii, testament en 1458) (23),

Antoine Aysaqui $(1460,1477)$

Michel de Prats (1477) (24),

Audebert David (1487) (25),

Guillaume Aysaqui (1489) (26),

Jean Le Bloy (1489) (27).

Un peu plus tard, nous voyons apparaître : Jean et Antoine Guononi $(1489,1510)(28)$,

Raymond Charmesson, 1509 (29),

Thomas David (1516) (30).

L'acte de mariage de Jean Moulin (31) (30 av. 1525) (fils de Jean) marque l'entrée dans le domaine mieux connu des dynasties modernes. Il y a donc incontestablement renouveau de l'activité ; ce renouveau attire les étrangers et il est en même temps entretenu par leurs apports. Pierre Badel vient d'Embrun, Pierre Armand de Langeac (diocèse de Saint-Flour), Jean Le Bloy est un Picard de Saint-Quentin en Vermandois. Dans le même temps, on constate un phénomène "d'émigra- tion " vers Aix qui témoigne à sa façon de l'existence d'une concentration de savoir-faire importante. L'émigration est peut-être un grand mot, mais il semble que certains potiers d'Apt se soient installés à Aix et ils y sont peut-être bien à l'origine d'une implantation artisanale qui, selon toute apparence, était inconnue dans la capitale de la Provence avant le XVe siècle. Antoine Galvi, olerius d'Apt, travaille à Aix en 1450 et 1451 , Antoine Gausii, aptésien en 1438 est aixois ou habitant de Puyricard en 1465, Thomas David et Jean Guononi travaillent à Aix au début du XVI e siècle (32). Cette expansion est probablement assez significative puisque, à compter de 1461, le conseil croit devoir désigner chaque année deux inspectores ollarum, qui deviennent en 1481, Inspectores gippi, tegularum et ollarum (33). Nous ignorons par contre si les relations commerciales suivent ; logiquement cela devrait être le cas, mais nous ne connaissons pour l'instant qu'un seul acte de vente de terrailles diverses par Antoine David à Guillerme Brune revendeuse d'Aix (34).

D'un point de vue pratique, que savons-nous des lieux et des modes de la production céramique aptésienne?

Bien peu de choses hélas. Quelques points cependant sont établis. Le quartier Saint-Lazare semble être un, sinon le lieu d'extraction de l'argile figuline. Monet Gausii en 1421 et 1427 et Jean Barralier en 1458 y ont leur terralh ou terrier. La vocation du quartier ne s'est pas démentie par la suite.

Il semble également qu'on ait pris de la terre près du chemin de Saint-Saturnin (1387). La situation des ateliers nous est inconnue sauf dans un cas (l'atelier de $\mathrm{M}$. Gausii est situé hors les murs "loco dicto in itinere quo itur ad fontem Duroni ".

Leur organisation est presque aussi mal connue. En 1412 et 1414 il est question de crottes (pièces voûtées ou grottes?); en 1458, Jean Barralier lègue sa boutique (dans laquelle il tourne), Monet Gausii loue son operatorium, dans lequel il tourne lui aussi. Il y a donc polyvalence du vocabulaire pour ne pas dire confusion. Le nombre de postes de travail est probablement de 1 ou 2. l en 1414,2 en 1427 et 1440 (35), ce qui est le lot commun à la même époque et plus tard dans nombre d'au-

(16) Ibid., Id., fo $310,324 \mathrm{v}^{\circ}, 23$ août 1425 et confirmation par la Ville.

(17) A.D. 84, Notaires d'Apt, Pondicq $144, f^{\circ} 3 v^{\circ}$ et 4.

(18) A.D. 13, annexe d'Aix, 302 E 292, f० $8 v^{\circ}, 26$ mai 1438 et $10 v^{\circ}, 11$ juin 1438.

(19) Ibid., Id., f० $8 \mathrm{v}^{\circ}$. Ibid.. $309 \mathrm{E} 181$, f० $71 \mathrm{v}^{\circ}, 25$ août 1446.

(20) Ibid., 302 E 292, fo 9, 3 juin 1438. A.C. Apt, BB 17, f० 108, 16 av. 1445. A.D. 84, Notaires d'Avignon, Martin 713, fo 142, 2 fév. 1446

(21) A.D. 84, Notaires d'Apt, Geoffroy $3, f^{\circ} 51 v^{\circ}, 3$ mars 1440.

(22) A.D. 13, annexe d'Aix, 309 E 123, 18 novembre 1450 et 306 E 226, 16 avril 1451.

(23) Ibid., 309 E 229, f० 48, 15 avril 1458.

(24) A.D. 13, B 973, f० $2 v^{\circ}, 24$ mars 1460. A.D. 13, annexe d’Aix, 302 E 545, f० 205, 21 av. 1477.

(25) A.D. 13, annexe d'Aix, 302 E 545, Partie registre factice, 7 juin 1487.

(26) A.D. 84, Notaires d'Apt, Geoffroy 37, fo 17 et $19 v^{\circ}, 26$ mars et 3 avril 1489.

(27) Ibid., Id., 1 fév. 1489

(28) Ibid., Id., f ${ }^{\circ} 4,15$ janvier 1489, Jean et Antoine Guononi. A.D. 13, annexe d'Aix, 306 E 444, 7 nov. 1510.

(29) A.D. 84, Notaires d'Avignon, étude antiq., N.B. de Jean Tavani, 10 déc. 1509.

(30) A.D. 13, annexe d'Aix, 308 E 935, f०260, 29 janvier 1516.

(31) A.D. 84, Notaires d'Apt, Geoffroy 87, f०215, 30 avril 1525.

(32) A.D. 13, Annexe d'Aix, 307 E 62, 23 juillet 1465.

(33) A.C. Apt, BB19, f० $1 v^{\circ}(27$ décembre 1461) : "Inspectores ollarum Audibertum Davidis et bartholomeum de Martino " fo 26 (29 décembre 1462). Les mêmes, f० 66 (29 décembre 1463): "Guilhermus senequerii et Bartholomeum Martini ". Ibid., BB 20, fo 28 (31 décembre 1481): "Inspectores gippi tegularum et ollarum Audebertus Davidis Johannes Clastroni ".

(34) A.D. 13, annexe d'Aix, 306 E 205, f० 179, 14 juin 1441.

(35) A.D. 84, Notaires d'Apt, Pondicq 144, fo $3 \mathrm{v}^{\circ}$ : " quoddam operatorium ollarum munitum duabus rotis fusteis... " Ibid., Id., Geoffroy $3, \mathrm{f}^{\circ} 51 \mathrm{v}^{\circ}, 3$ mars 1440 : "Duas rotas..." ". 
tres centres. Le plus intéressant mais pas le plus étonnant est sans doute l'existence d'ateliers sans fours. L'atelier de Monet Gausii n'en a pas, pas plus que celui de Bertrand Faraud. A l'inverse, il existe des fours isolés appartenant à des non potiers; deux cas en 1440. Cela nous amène tout naturellement à poser le problème de l'organisation de la production et du statut du potier. Nous disposons de très peu d'éléments pour l'apprécier. Il y a évidemment une hiérarchie qui s'organise du Maître, propriétaire de son atelier, à l'apprenti, en passant par les niveaux intermédiaires de l'operarius (Pierre Armand) ou du famulus (chez Ant. Galvi), mais le plus souvent les nuances ne sont pas de mise, on est désigné comme figulus ou ollerius et il est difficile de distinguer ainsi le maître et l'employé. Le statut le mieux connu est toujours, et paradoxalement, le moins enviable, celui d'apprenti. Les contrats aptésiens sont dans la norme; ils varient de 2 à 5 ans, parfois plus. Le maitre doit l'enseignement, la protection et la nourriture. Parfois des chaussures ou un autre avantage en nature.

A la périphérie du monde des artisans, on trouve parfois trace de travailleurs occasionnels ou à temps partiel qui sont surtout des polyvalents, tel ce barbier qui vend des marmites crues à un potier en 1427 (36). Avec eux se pose le problème de la spécialisation (qui n'est certes pas nouveau). Enfin, de tous les aspects de l'artisanat céramique le plus difficile à cerner est sans contexte le produit lui-même. Nous n'en savons rien avant le XVIe siècle car on ne le nomme ni ne le différencie. Le seul terme employé est oulle qui peut désigner n'importe quoi, c'est ainsi que, par exemple, Ant.
David s'engage à livrer des oulles de tout obrage. Les objets en terre cuite sont comptés en nombre (terme encore en vigueur au $\mathrm{XIX}^{\mathrm{e}}$ siècle désignant un assortiment d'importance variable convenu entre fabricant et acheteur). Mais il n'est pas exclu qu'il ait pu avoir ici ou là une valeur fixe. On distingue dans un cas (1441) des nombres d'obrages doble dont nous ignorons ce qu'ils sont et nous connaissons la valeur en nombres (6) d'une saumée d'oulles au XVe siècle, c'est-à-dire la charge d'une bête de somme.

Si nous prenons quelque hauteur, pour conclure sur ce point des balbutiements de l'artisanat céramique à Apt, tels que les sources écrites nous les font connaître, l'ancienneté de l'industrie céramique aptésienne paraît certaine, plus de six siècles au travers des seuls textes. Mais son importance n'est pas égale au fil de cette longue séquence chronologique; le redémarrage consécutif à la crise du XIVe siècle ne doit pas masquer une certaine médiocrité, qui doit se comprendre au sens étymologique. Il est difficile au XVe siècle au moins de comparer Apt à d'autres centres plus importants et certainement plus productifs. Un exemple le montre bien, celui de la Leyde de Carpentras. Les tarifs taxent effectivement les poteries de Diculefit, de Saint-Quentin dans l'Uzège, ainsi que celles de Bédoin, mais il n'est pas fait mention de celles d'Apt. Sa notoriété viendra plus tard, soutenue par des facteurs objectifs : la terre d'Apt devait avoir de singulières qualités pour qu'en 1531 un potier en fasse venir 18 quintaux de la variété dite Blanque jusqu'à Aubagne (37).

(36) Ibid., Id., Pondicq 144, f० 4, 4 février 1427. Pons de Ranco, barbier d'Apt, a vendu à Pierre Badel : "... quindecim duodenarum et medie operis ollarum crudarum..." ".

(37) A. d'AGNEL, La Faïence et la porcelaine de Marseille, 1911, p. 27. "... Duodecim quintalia terre blanque d'Apt..." "... alia sex quintalia predicte terre". 